\title{
Distributed Terminal Backstepping control for Multi-Agent Euler-Lagrange Systems
}

\author{
Hu Chen \\ Dep. of Electronic Eng., Pusan National University, Jangjeon-dong, Geumjeong-gu \\ Busan, 609-735,Korea Republic \\ Seong-Ik Han \\ Dep. of Electronic Eng., Pusan National University, Jangjeon-dong, Geumjeong-gu \\ Busan, 609-735,Korea Republic \\ Jang-Myung Lee \\ Dep. of Electronic Eng., Pusan National University, Jangjeon-dong, Geumjeong-gu \\ Busan, 609-735,Korea Republic \\ E-mail: chenhu@pusan.ac.kr,skhan@pusan.ac.kr,jmlee@pusan.ac.kr \\ www.pusan.ac.kr
}

\begin{abstract}
This paper presents a distributed terminal (finite-time) backstepping consensus control for multi-agent EulerLagrange systems. Terminal virtual error surfaces and virtual controls are proposed to guarantee the finite-time error consensus and formation convergence of a group of one-leader and multi-follower cooperative tracking EulerLagrange system.Finite-time stability including infinite-time stability was proved by the finite-time Lyapunov candidate function. Simulation example shows the effectiveness of the proposed finite-time backstepping coordinated tracking controller.
\end{abstract}

Keywords: Euler-Lagrange multi-agent system, backstepping control, Terminal virtual error surface.

\section{Introduction}

In recent years, there has been a great interest for researches of multi-agent systems, whose applications include spacecraft, mobile robots, sensor networks, etc. Interesting research directions are containment control, consensus, formation, and flocking control [1]. These problems focus on two cases, namely, the case that there does not exist a leader and the case where there exists a leader. The coordinate tracking problems to track a single leader have been investigate for followers with single-integrator, double-integrator, high-order dynamics, nonlinear or Euler-Lagrange dynamics [2-5]. Linear control theory and variable structure control methods in most researches are used. On the other hand, there are few examples that use the backstepping control technique [6] for nonlinear or Euler-Lagrange multi-agent system. In this method,the problem of unmatched uncertainty and neglecting the efficient nonlinearities is overcomevia adopting step-bysteprecursiveprocess.

However,although a controller designed using this theorem guarantees the infinite-time stability of a closed-loop system, it has drawbacks such as a slow convergence rate and reduced robustness to uncertainty. On the other hand, systems with finite-time settlingtime design possess attractive features such as improved robustness and disturbance rejection properties [7],In this paper, terminal backstepping control based multi-agent consensus control for EulerLagrange system with one-leader and multi-followers is developed.

\section{Background and Preliminaries}

\subsection{Concept of Graph Theory}

In this paper, multi-agent robot Euler-Lagrange systems consisting of one leader and $n$ followers are considered. 
Graph theory is introduced to solve the coordination problem and model information exchange between agents. The communication topology is a directed graph, $\mathcal{G}=\{\mathscr{V}, \mathcal{E}\}$, where $\mathcal{V}=\{0,1,2, \ldots, \mathrm{n}\}$ is the set of nodes, node $i$ represents the $i$ th agent, $\mathcal{E}$ is the set of edges, and an edge in $\mathcal{G}$ is denoted by an ordered pair $(i, j) .(i, j) \in \mathcal{E}$ if and only if the $i$ th agent can send information to $j$ th agent directly, but not necessarily vice versa.A directed tree is a directed graph, where every node has exactly one parent except for the root, and the root has directed paths to every other node. A directed graph , $\mathcal{G}=\{\mathcal{V}, \mathcal{E}\}$, has a directed spanning tree if and only if $\{\mathscr{V}, \mathcal{E}\}$ has at least one node with a directed path to all other nodes. $A=\left[a_{i, j}\right] \in R^{(n+1) \times(n+1)}$ is called the weighted adjacency matrix of $\mathcal{G}$, where $a_{i i}=0$ and $a_{i j} \geq 0$ with $a_{i j}>0$ if there is an edge between the $i$ th agent and $j$ th. The Laplacian of the weighted graph can be defined as $L=D-A \in R^{(n+1) \times(n+1)} \quad$,where $D=\operatorname{diag}\left(d_{0}, d_{1}, \ldots, d_{n}\right) \in R^{(n+1) \times(n+1)}$ is the degree matrix and $d_{i}=\sum_{j=0}^{n} a_{i j}$ for $i=0,1, \ldots, n$. For simplicity, it is assumed that $a_{i j}=1$ if $(i, j) \in \mathcal{E}$ and 0 otherwise. The connection weight between agent $i$ and the leader is denoted by $b_{i}$ such that $b_{i}=1$ if agent $i$ connected to the leader and 0 otherwise.

\subsection{Multi-Agent Euler-Lagrange Systems}

The nonlinear dynamics of a group of $n+1$ fully actuated Euler-Lagrange systems are described as follows:

$M_{i}\left(q_{i}\right) \ddot{q}_{i}+C_{i}\left(q_{i}, \dot{q}_{i}\right) \dot{q}_{i}+G_{i}\left(q_{i}\right)+\tau_{d i}=\tau_{i}, i=1, \ldots, n+1$,

where $M_{i}\left(q_{i}\right)$ is a symmetric and positive definite inertia matrix; $C_{i}\left(q_{i}, \dot{q}_{i}\right)$ is a velocity-dependent centripetal and Coriolis forcesmatrix; $G_{i}\left(q_{i}\right)$ is a gravitational vector; $\tau_{d i}$ is a bounded unknown disturbance including unmodelled dynamics and exogenous disturbance; and $\tau_{i}$ is an input torque. The simple dynamic equation can be expressed as the following state space model:

$$
\begin{aligned}
& \dot{x}_{i, 1}=x_{i, 2}, \\
& \dot{x}_{i, 2}=f_{i}\left(\bar{x}_{2}\right)+g_{i}\left(\bar{x}_{2}\right) u_{i}, \\
& y_{i}=x_{i, 1}, i=1, \ldots, n+1,
\end{aligned}
$$

where $x_{i, 1}=q_{i}, x_{i, 2}=\dot{q}_{i}, \bar{x}_{2}=\left[x_{i, 1}, x_{i, 2}\right]^{T}, f_{i}\left(\bar{x}_{2}\right)=$ $-M_{i}^{-1} C_{i}\left(\bar{x}_{i, 2}\right) x_{i, 2} \quad-M_{i}^{-1} G_{i}\left(x_{i, 1}\right)-M_{i}^{-1} \tau_{d i}, g_{i}=M_{i}^{-1}$, and $u_{i}=\tau_{i}$.

Assumption 1. $\left\|M_{i}^{-1} \tau_{d i}\right\| \leq \delta_{d i},\left\|K_{i}^{C}-M_{i}^{-1} C_{i}\left(\bar{x}_{i, 2}\right)\right\| \leq \delta_{c i}$,
$\left\|K_{i}^{G} X_{i, 1}-M_{i}^{-1} G_{i}\left(x_{i, 1}\right)\right\| \leq \delta_{g i} \quad, \quad$ and $\quad \delta_{c i}+\delta_{g i}+\delta_{d i} \leq \delta_{h i} \quad$, where $K_{i}^{C}$ and $K_{i}^{G}$ are positive definite diagonal matrices and vectors, respectively, and $\delta_{h i}>0$ are upper bounds.

\section{Distributed Terminal backstepping Controller Design and Stability Analysis}

\subsection{Controller Design}

The tracking errorsand virtual error surfaces are defined as follows:

$$
\begin{aligned}
& z_{i, 1}=\sum_{j=1}^{n} a_{i j}\left(y_{i}-y_{j}\right)+b_{i}\left(y_{i}-x_{0}\right), \\
& z_{i, 2}=x_{i, 2}+c_{i, 1} \operatorname{sig}\left(z_{i, 1}\right)^{\gamma_{i, 1}}-\alpha_{i, 1}, i=1, \ldots, n,
\end{aligned}
$$

where $x_{0}$ is the position of the leader, $\alpha_{i, 1}$ are the virtual controls, $\quad \operatorname{sig}\left(z_{i, 1}\right)=\left\|z_{i, 1}\right\|^{\gamma_{i, 1}} \operatorname{sgn}\left(z_{i, 1}\right) \quad, \quad c_{i, 1}>0 \quad$ are constants, and $\gamma_{i, 1}=\xi_{i, 1} / \zeta_{i, 1}, \xi_{i, 1}$ and $\zeta_{i, 1}$ are positive odd numbers, $\xi_{i, 1}<\zeta_{i, 1}<2 \xi_{i, 1}, \operatorname{sgn}\left(z_{i, 1}\right)$ is a $\operatorname{sign}$ function,. (3) can be changed for the formation control case as follows:

$$
z_{i, 1}=\sum_{j=1}^{n} a_{i j}\left(y_{i}+\Delta_{i}-y_{j}-\Delta_{j}\right)+b_{i}\left(y_{i}+\Delta_{i}-x_{0}-\Delta_{0}\right)
$$

The time derivative of the first error surfaces $z_{i, 1}$ along (2) is

$$
\begin{gathered}
\dot{z}_{i, 1}=\left(d_{i}+b_{i}\right)\left(z_{i, 2}-c_{i, 1} \operatorname{sig}\left(z_{i, 1}\right)^{\gamma_{i, 1}}+\alpha_{i, 1}\right) \\
-\sum_{j=1}^{n} a_{i j} x_{i, 2}-b_{i} \dot{x}_{0} .
\end{gathered}
$$

The Lyapunov function candidate $V_{i, 1}=z_{i, 1}^{T} z_{i, 1} / 2$ to design the distributed virtual controller. Differentiating $V_{i, 1}$ yields

$$
\begin{aligned}
\dot{V}_{i, 1}= & z_{i, 1}^{T}\left[\left(d_{i}+b_{i}\right)\left(z_{i, 2}-c_{i, 1} \operatorname{sig}\left(z_{i, 1}\right)^{\gamma_{i, 1}}+\alpha_{i, 1}\right)\right. \\
& \left.-\sum_{j=1}^{n} a_{i j} x_{i, 2}-b_{i} \dot{x}_{0}\right] .
\end{aligned}
$$

Choosing the distributed virtual control as

$$
\alpha_{i, 1}=\frac{1}{d_{i}+b_{i}}\left(-k_{i, 1} z_{i, 1}+\sum_{i=1}^{n} a_{i j} x_{i, 2}+b_{i} \dot{x}_{0}\right),
$$

(7) becomes

$$
\dot{V}_{i, 1}=-k_{i, 1} z_{i, 1}^{T} z_{i, 1}-\left(d_{i}+b_{i}\right) c_{i, 1}\left\|z_{i, 1}\right\|^{\gamma_{i, 1}+1}+\left(d_{i}+b_{i}\right) z_{i, 1}^{T} z_{i, 2},
$$

where $k_{i, 1}>0$ are constants. Differentiating the Lyapunov function

$$
\begin{aligned}
V_{i, 2}= & V_{i, 1}+z_{i, 2}^{T} z_{i, 2} / 2+\tilde{\delta}_{h i}^{2} / 2 \eta_{i} \text {, along (2) and (4), } \\
\dot{V}_{i, 2}= & -k_{i, 1} z_{i, 1}^{T} z_{i, 1}-\left(d_{i}+b_{i}\right) c_{i, 1}\left\|z_{i, 1}\right\|^{\gamma_{i, 1}+1}+\left(d_{i}+b_{i}\right) z_{i, 1}^{T} z_{i, 2} \\
& +z_{i, 2}^{T}\left[f_{i}\left(\bar{x}_{2}\right)+g_{i}\left(\bar{x}_{2}\right) u_{i}+c_{i, 1} \gamma_{i, 1}\left\|z_{i, 1}\right\|^{\gamma_{i, 1}-1} \dot{z}_{i, 1}-\dot{\alpha}_{i, 1}\right] \\
& -\tilde{\delta}_{h i} \dot{\hat{\delta}}_{h i} / \eta_{i} .
\end{aligned}
$$

Choosing the control inputs and adaptive laws as 


$$
\begin{aligned}
u_{i} & =g_{i}^{-1}\left[-k_{i, 2} z_{i, 2}-\left(d_{i}+b_{i}\right) z_{i, 1}^{T}+K_{i}^{C} x_{i, 2}+K_{i}^{G} x_{i, 1}\right. \\
& -c_{i, 1} \gamma_{i, 1}\left\|z_{i, 1}\right\|^{\gamma_{i, 1}-1} \dot{z}_{i, 1}-c_{i, 2} \operatorname{sig}\left(z_{i, 2}\right)^{\gamma_{i, 2}} \\
& \left.+\frac{\hat{\delta}_{h i} z_{i, 2}}{\left\|z_{i, 2}\right\|+\kappa_{i, 2}}+\dot{\alpha}_{i, 1}\right], \\
\dot{\hat{\delta}}_{h i} & =\eta_{i}\left(\left\|z_{i, 2}\right\|^{2} /\left(\left\|z_{i, 2}\right\|+\kappa_{i, 2}\right)-\eta_{i}^{\prime} \hat{\delta}_{h i}\right),
\end{aligned}
$$

where $k_{i, 2}>0, \eta_{i}>0, \eta_{i}^{\prime}>0, c_{i, 2}>0$, and $0.5<\gamma_{i, 2}<1$ are constants, $\tilde{\delta}_{h i}=\delta_{h i}-\hat{\delta}_{h i}, \hat{\delta}_{h i}$ are estimates of $\delta_{h i}$, we obtain the following expression:

$$
\begin{aligned}
\dot{V}_{i, 2} \leq & -\sum_{k=1}^{2} k_{i, 1} z_{i, k}^{T} z_{i, k}-\left(d_{i}+b_{i}\right) c_{i, 1}\left\|z_{i, 1}\right\|^{\gamma_{i, 1}+1}-c_{i, 2}\left\|z_{i, 2}\right\|^{\gamma_{i, 2}+1} \\
& +\tilde{\delta}_{h i}\left(z_{i, 2}^{T}-\hat{\delta}_{h i} / \eta_{i}\right) \\
\leq & -\sum_{k=1}^{2} k_{i, 1} z_{i, k}^{T} z_{i, k}-\frac{\eta_{i}^{\prime} \tilde{\delta}_{i}^{2}}{2}-\sum_{k=1}^{2} \beta_{i, k}\left\|z_{i, k}\right\|^{\gamma_{i, k}+1}+\eta_{i}^{\prime} \delta_{i}^{2} / 2 \\
\leq & -\left(\sum_{k=1}^{2} k_{i, k} z_{i, k}^{T} z_{i, k}+\frac{\eta_{i}^{\prime} \tilde{\delta}_{i}^{2}}{2}\right)-\left(\sum_{k=1}^{2} \beta_{i, k} z_{i, k}^{T} z_{i, k}+\frac{\eta_{i}^{\prime} \tilde{\delta}_{i}^{2}}{2}\right)^{\frac{\gamma_{i, k}+1}{2}} \\
& \quad+\mu_{i} \\
\leq & -a V_{i, 2}-b^{\frac{\gamma_{i, k}+1}{2}} V_{i, 2}^{\frac{\gamma_{i, k}}{2}}+\mu_{i},
\end{aligned}
$$

where $\beta_{i, k}=\min \left[\left(d_{i}+b_{i}\right) c_{i, 1}, c_{i, 2}\right], a=\min \left[2 k_{i, 1}, 2 k_{i, 2}, \eta_{i}^{\prime}\right]$,

$$
b=\min \left[2 \beta_{i, 1}, 2 \beta_{i, 2}, \eta_{i}^{\prime}\right], \mu_{i}=\eta_{i}^{\prime}\left\|z_{i, k} \tilde{\delta}_{i}\right\|^{2}+\left(\frac{\eta_{i}^{\prime} \tilde{\delta}_{i}^{2}}{2}\right)^{2}+\frac{\eta_{i}^{\prime} \delta_{i}^{2}}{2}
$$

\subsection{Finite-Time Stability Analysis}

((13) can be rewritten the following two forms:

$$
\begin{aligned}
& \dot{V}_{i, 2} \leq-a V_{i, 2}-\left(b^{\frac{\gamma_{i, k}+1}{2}}-\frac{\mu_{i}}{V_{i, 2}^{\frac{\gamma_{i, k}+1}{2}}}\right) V_{i, 2}^{\frac{\gamma_{i, k}+1}{2}} \\
=- & a^{\prime} V_{i, 2}-b^{\prime} V_{i, 2}^{\gamma^{\prime}}
\end{aligned}
$$

where $a^{\prime}=a-\mu_{i} / V_{i, 2} \quad, \quad b^{\prime}=b^{\frac{\gamma_{i, k}+1}{2}}-\mu_{i} / V_{i, 2}^{\frac{\gamma_{i, k}+1}{2}}$, and $\gamma^{\prime}=\frac{\gamma_{i, k}+1}{2}$.From (14), if $a$ and $b$ is selected such that $a>\mu_{i} / V_{i, 2}$ and $b>\mu^{\frac{2}{\gamma_{i, k}+1}} / V_{i, 2}$, respectively. Then, from the definition of finite-time stability [7], the equilibrium point $x=0$ is globally finite-time stable and the settling time $t_{s}$ can be given by

$$
t_{s} \leq \frac{1}{a\left(1-\gamma^{\prime}\right)} \ln \frac{a V^{1-\gamma^{\prime}}\left(x_{0}\right)+b^{\prime}}{b^{\prime}} .
$$

\section{Simulation Example}

To validate the proposed control scheme, the following group of one leader indexed by 0 and four followers indexed by 1, 2, 3, and 4, respectively as shown in Fig. 1. The strict feedback state equations of each agent are expressed as

$$
\begin{aligned}
& x_{i, 1}=x_{i, 2}, \\
& x_{i, 2}=f_{i, 2}\left(x_{i, 1}\right)+g_{i, 2} u_{i},
\end{aligned}
$$

where $f_{i, 2}=-\left[G_{i}\left(q_{i}\right)+\tau_{d i}\right] / J_{i}, g_{i}=1 / J_{i}, u_{i}=\tau_{i}$ $J_{i}=m_{i} L_{i}^{2} / 3, G_{i}\left(q_{i}\right)=m_{i} L_{i} \cos q_{i}$, the mass of the link $m_{i}=1 \mathrm{~kg}$, and the length of link $L_{i}=0.25 \mathrm{~m}$. Let the initial condition of four followers be $x_{1,1}=1, x_{1,2}=0$, $x_{2,1}=1.2, x_{2,2}=0, x_{3,1}=2, x_{3,2}=0, x_{4,1}=-1.2, x_{4,2}=0$. The Laplacian can be written as

$$
L=\left[\begin{array}{ccccc}
0 & 0 & 0 & 0 & 0 \\
0 & 3 & -1 & -1 & -1 \\
0 & 0 & 1 & -1 & 0 \\
-1 & 0 & -1 & 1 & 0 \\
-1 & 0 & 0 & 0 & 0
\end{array}\right], b_{3}=1, b_{4}=1
$$

Simulation results are obtained with the time-varying control input to the leader being designed as $u_{0}=-\sin \left(x_{0,1}\right) /\left(1+e^{-t}\right), x_{0,1}=\pi / 2$, and $x_{0,2}=0$.

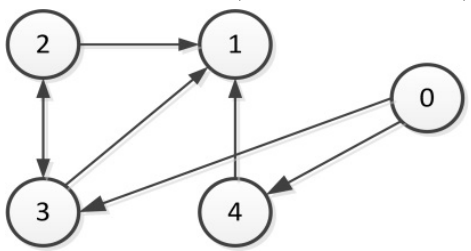

Fig. 1. Directed graph of the manipulator group The error functions for the illustration of the formation) control are changed into (5), where $\Delta_{1}=-1, \Delta_{2}=-2, \Delta_{3}=-3$, and $\Delta_{4}=-4$. Simulation results are presented in Fig. 2 (consensus control) and Fig. 3 (formation control), where the settling time of the proposed TBSC system is $31 \%$ faster than that of the BSC system. In addition, the steady state errors of the TBSC system are smaller compared to the BSC system.
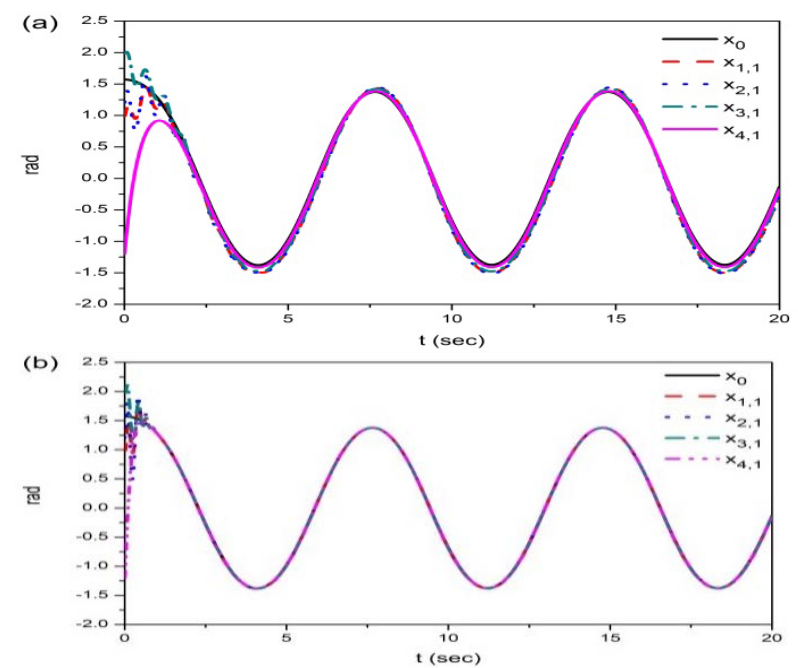

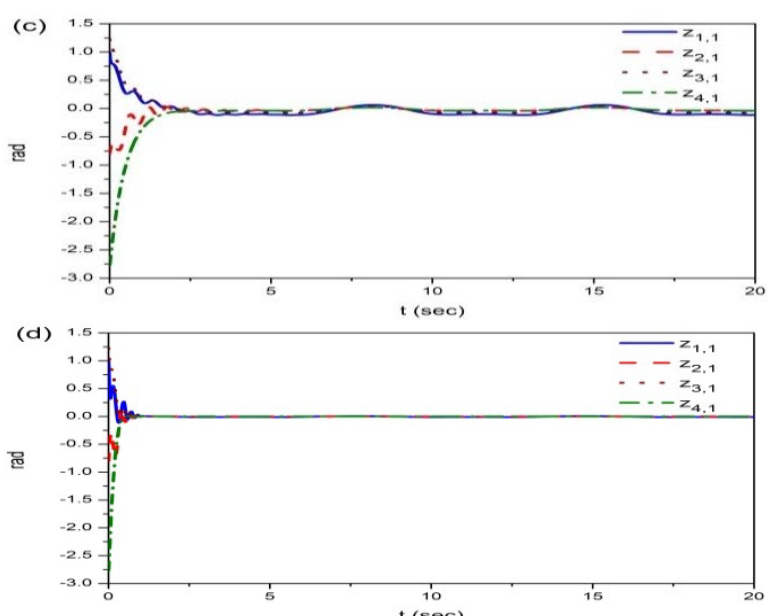

Fig. 2. Consensus control simulation results. (a) Tracking outputs of BSC system. (b) Tracking outputs of TBSC system. (c) $z_{1,1}$ of BSC. (d) $z_{1,1}$ of TBSC.
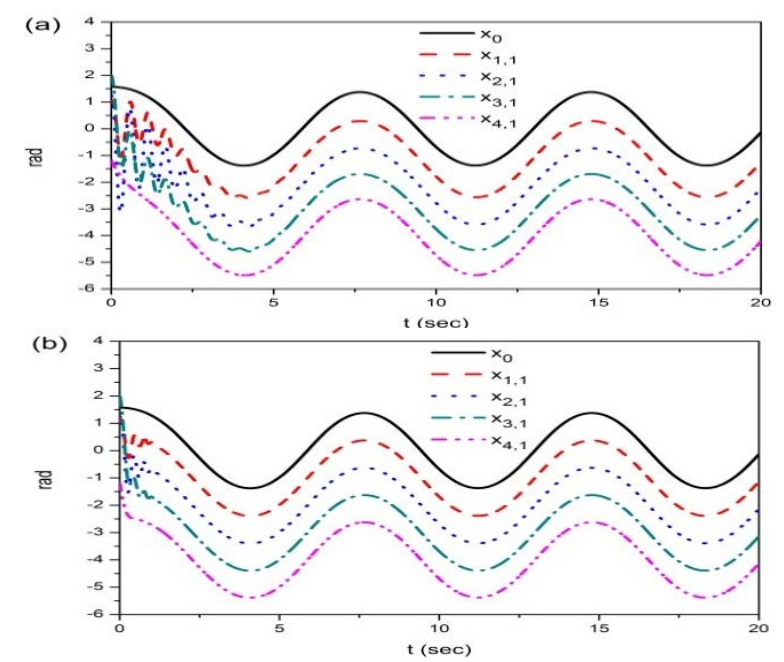

Table 1. Settling time (sec) of BSC and TBSC systems

\begin{tabular}{ccccc}
\hline & \multicolumn{2}{c}{ Consensus } & \multicolumn{2}{c}{ Formation } \\
\cline { 2 - 5 } & BSC & TBSC & BSC & TBSC \\
\hline$z_{1,1} \leq 0.01$ & $2.03 \mathrm{~s}$ & $0.77 \mathrm{~s}$ & $2.23 \mathrm{~s}$ & $0.83 \mathrm{~s}$ \\
$z_{2,1} \leq 0.05$ & $2.20 \mathrm{~s}$ & $0.67 \mathrm{~s}$ & $2.63 \mathrm{~s}$ & $0.91 \mathrm{~s}$ \\
$z_{3,1} \leq 0.01$ & $2.07 \mathrm{~s}$ & $0.76 \mathrm{~s}$ & $2.83 \mathrm{~s}$ & $0.86 \mathrm{~s}$ \\
$z_{4,1} \leq 0.05$ & $2.15 \mathrm{~s}$ & $0.42 \mathrm{~s}$ & $1.46 \mathrm{~s}$ & $0.29 \mathrm{~s}$ \\
Mean (\%) & $100 \%$ & $32 \%$ & $100 \%$ & $31 \%$ \\
\hline
\end{tabular}

\section{Conclusion}

A terminal backstepping control scheme to guarantee the fast error convergence and small tracking error performance for a multi-agent Euler-Lagrange system is developed in this paper. A virtual finite-time error surface is defined to design a virtual control. The finitetime convergence is proved by the finite-time stability analysis of Lyapunov function. Simulation for one-link manipulator agents confirms the theoretical proposal.

\section{Acknowledgements}

This research was supported by the MOTIE (Ministry of Trade, Industry \& Energy), Korea, under the Industry Convergence Liaison Robotics Creative Graduates Education Program supervised by the KIAT (N0001126).

This work was supported by the National Research Foundation of Korea(NRF) Grant funded by the Korean Government(MSIP) ( NRF-2013R1A1A2021174 )

This research was supported by the MOTIE (Ministry of Trade, Industry \& Energy), Korea, under the Industry Convergence Liaison Robotics Creative Graduates Education Program supervised by the KIAT (N0001126)

\section{References}

1. W. Ren, Distributed consensus in multi-vehicle cooperativecontrol (Springer-Verlag, London,2008).

2. H. Arcak, and M. Wen, Adaptive motion coordination: using relative velocity feedback to track a reference velocity, Automatica, 45(2) (2009) 1020-1025.

3. H. Su, G. Chen, and Z. Lin, Adaptive second-order consensus of networked mobile agents with nonlinear dynamics, Automatica, 47(2) (2011) 368-375

4. H. Zhang, and F. L. Lewis, Adaptive cooperative tracking control of high-order nonlinear systems with unknown dynamics, Automatica, 48 (2012) 1432-1439.

5. S. Chung, and J. J. Slotine, Cooperative robot control and concurrent synchronization of Lagrangian systems, IEEE Tran. Robotics, 25(3) (2012) 686-700.

6. K. Kristic, I.Kanellakopoulos, and P. V. Kokotovic, Nonlinear and adaptive control design (Wiley, New York, 1995).

7. S. Yu, X. Yu, B. Shirinzadeh, and Z. Man, Continuous finite-time control for robotic manipulators with terminal sliding mode, Automatica, 41(2005)1975-1964. 